\title{
www.czasopisma.pan.pl \\ ThermoCalc Application for the Assessment of Binary Alloys Non-Equilibrium Solidification
}

\author{
A. Zyska *, Z. Konopka, M. Lągiewka, P. Kordas \\ Foundry Department, Czestochowa University of Technology, \\ Al. Armii Krajowej 19, 42-200 Częstochowa, Poland \\ *Corresponding author. E-mail address: zyska@wip.pcz.pl
}

Received 07.07.2016; accepted in revised form 30.07.2016

\begin{abstract}
The paper presents the possibility of application of the developed computer script which allows the assessment of non-equilibrium solidification of binary alloys in the ThermoCalc program. The script makes use of databases and calculation procedures of the $P O L Y-3$ module. A solidification model including diffusion in the solid state, developed by Wołczyński, is used to describe the non-equilibrium solidification. The model takes into account the influence of the degree of solute segregation on the solidification process by applying the so-called back-diffusion parameter. The core of the script is the iteration procedure with implemented model equation. The possibility of application of the presented calculation method is illustrated on the example of the $\mathrm{Cr}-30 \% \mathrm{Ni}$ alloy. Computer simulations carried out with use of the developed script allow to determine the influence of the back-diffusion parameter on the course of solidification curves, solidus temperature, phase composition of the alloy and the fraction of each phase after the solidification completion, the profile of solute concentration in liquid during solidification process, the average solute concentration in solid phase at the eutectic temperature and many other quantities which are usually calculated in the ThermoCalc program.
\end{abstract}

Keywords: ThermoCalc, Non-equilibrium solidification, Segregation, Wołczyński model, Binary alloys

\section{Introduction}

The presently used computer programs dedicated for thermodynamic calculations and creation of phase diagrams for multicomponent systems apply the CALPHAD method [1]. The concept of this method is bases on the mathematical description of the system phases (gas, liquid, and solid phases) by the thermodynamic functions and on minimization of Gibbs free enthalpy. In the calculation procedure, the total free enthalpy of the individual phases of the system is represented by the sum of enthalpies of pure components, the enthalpy of mixing for the ideal solution, and the excess enthalpy of solid solution mixing process [1-4].
In the case of metal alloys, their phase components usually produce solid solutions in wide range of chemical compositions. Therefore the form of Gibbs function applied to describe metallic solutions is of particular importance. It influences the accuracy of phase equilibrium calculations and the shape of achieved phase diagrams. The CALPHAD method applies the regular and subregular solution models taking into account the Redlich-Kister approximation [5-7]. The main advantage of this approach is the possibility of predicting the configuration of phase equilibrium systems in complex systems on the basis of thermodynamic parameters determined for binary systems.

The CALPHAD method replaced the conventional methods of construction of phase diagrams because it allowed for precise 
computing of phase equilibria for various materials with complex chemical compositions. Calculation of phase diagrams, however, demands a highly accurate thermodynamic data. Collecting, proceeding and verification of data concerning the thermodynamic properties, as well as computing the equilibrium diagrams for metal alloys, slags, ceramic materials, melted salts, aqueous solutions, etc. is the task of large consortia uniting the leading research centres. The world standard for commercial programs for thermodynamic calculations are the databases built by the SGTE consortium (Scientific Group Thermodata Europe). Some databases are built to order for companies producing articles made of modern technical materials, but these are generally not available for public use.

One of the commercial programs based on the CALPHAD approach is ThermoCalc, which was applied in the presented work. The program has modular structure, briefly presented in Figure 1.

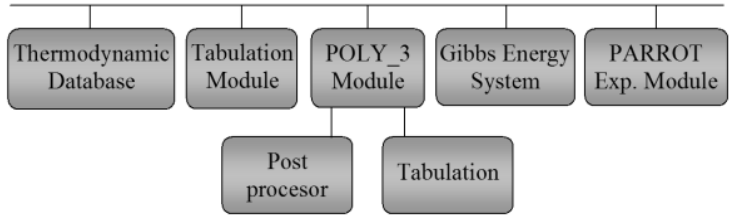

Fig. 1. Modules of thermodynamic program ThermoCalc [8]

The basic modules are the thermodynamic databases and the POLY 3 module dedicated to calculate phase equilibria by means of numerical methods. The GES module (Gibbs Energy System) is used to develop Gibbs function models, and the PARROT module serves to optimize thermodynamic parameters on the basis of experimental results. The tabulation module gives the possibility of printing parameters and thermodynamic quantities in a tabular form, and the Post processor allows for creating the graphic representation of calculation results.

ThermoCalc program is applied for quick analysis of solidification and the following phase transformation proceeding in solid state in alloys of complex chemical composition, and in particular [8-13]:

- to determine the liquidus and solidus temperature;

- to work out the solidification curves for equilibrium conditions;

- to assess the microsegregation of alloying elements when solidification takes place under the condition that no back-diffusion occurs in the solid state (Scheil model);

- to calculate the latent heat of solidification and the specific heat of alloys;

- to determine phase transition points in the solid state;

- to calculate the changes in phase composition of the alloy, the weight fractions of individual phases and their chemical composition as a function of temperature.

Despite its various possibilities and the wide range of application, the ThermoCalc program allows to assess the solidification of metal alloys only under the equilibrium conditions or when the segregation with no solid state diffusion takes place. However, the global equilibrium state cannot be achieved during the real solidification of a casting so that the degree of segregation of the alloying elements and the number and types of phases occurring in the structure of a solidified casting depend additionally on the rate of heat removal and the rate of diffusion. To take into account these processes and to model the phase composition of an alloy, it is necessary to couple the ThermoCalc program calculations with those of DICTRA program, which solves the diffusion equation [14].

This paper presents a part of a comprehensive research study covering the modelling of the non-equilibrium solidification of multi-component alloys with use of ThermoCalc program. One of the sub-targets of this work was to work out the computer executable script making use of the databases and numerical procedures (iterations) of the ThermoCalc program, which would enable the prediction of phase compositions in binary alloys solidifying with back-diffusion in the solid state. The Wołczyński model was used to describe the non-equilibrium solidification.

\section{Model of solidification including the solid state diffusion}

The problem of solidification of binary alloys with partial segregation of the solute was described in many theoretical works [15-19]. A number of analytical solutions is available, but particular attention should be paid to the universal model developed by Wołczyński [18], which introduces only small number of simplifications. The versatility of this model allows for simple implementation to the calculation procedures of ThermoCalc program, as well as for applying it in the case of the non-equilibrium solidification of binary or other multicomponent alloys. The segregation of the solute in this model is considered within a volume element of the length $L$ (Fig. 2), inside which the solidification front is moving in the $x$ direction at the rate $u$. The momentary position of the solidification front in the volume element determines the total fraction of the solid phase $f_{f}=x_{f} / L$, and the distance $x\left(0 \leq x_{S} \leq x_{f}\right)$ measured inside the crystal is related to the fraction $f_{S}=x / L$. To describe the solidification in a form of function, it was assumed that the solute diffusion occurs in the solid phase, and the liquid is uniform with respect to the solute concentration. The detailed description of the model can be found in References [18-20].

As a solution it was found an equation describing the change in the solute concentration in liquid phase as a function of the solid state fraction and the so-called back-diffusion coefficient:

$C_{L}\left(f_{S}, \alpha\right)=C_{0}\left[1-\left(1-\alpha k_{0}\right) f_{S}\right]^{\frac{k_{0}-1}{1-\alpha k_{0}}}$

where: $f_{\mathrm{S}} \in\left[0, f_{\mathrm{f}}\right], \quad f_{f} \in\left[0, f_{\mathrm{k}}\right], \alpha \in[0,1] ; C_{0}-$ solute concentration in the alloy, $k_{0}$ - equilibrium solute partition coefficient, $f_{\mathrm{k}}$ - critical fraction of liquid phase at which the liquid achieves characteristic concentration, which depends on the type of phase equilibrium system and is determined either by the eutectic, or peritectic, or else monotectic point concentration $-C_{\mathrm{E}}$, $C_{\mathrm{P}}$, and $C_{\mathrm{M}}$, respectively; $\alpha$ - the back-diffusion parameter which can be determined by experimental measurements with respect to segregation $[18,19]$, defined as:

$\alpha=\frac{D_{S}}{L u}=\frac{D_{S} t_{l}}{L^{2}}=\frac{t_{l}}{t_{h}}$ 
where: $D_{\mathrm{S}}$ - solute diffusion coefficient in the solid phase, $t_{1}=L / u$ - local solidification time, $t_{\mathrm{h}}=L^{2} / D_{\mathrm{S}}$ - time of total homogenization of solute concentration in solid phase.



Solid fraction, $f_{\mathrm{S}}$

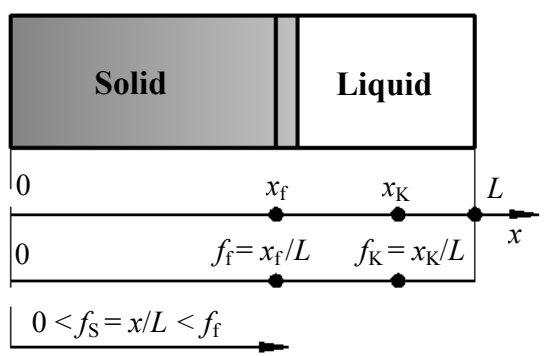

Fig. 2. The scheme of crystal growth in the volume element of the length $L$; designations: $C_{0}, C_{\mathrm{C}}\left(f_{\mathrm{S}}, \alpha\right)-$ initial and actual concentration of solute in liquid, $\bar{C}_{S}\left(f_{S}, \alpha\right)$ - the average concentration of solute in solid phase, $C_{\mathrm{S}}\left(f_{\mathrm{s}}, \alpha\right)$ - the 'history' of solute concentration in the solid state at the crystallization front

The back-diffusion parameter describes the solute segregation in solid phase and can take the values from within the 0 to 1 range. When $\alpha=0$, there is no diffusion in the solid state and the equation (1) is simplified to the Scheil equation. For $\alpha=1$, the solute diffusion in solid phase proceeds at the infinite rate, and the equation (1) is simplified to the equation describing the equilibrium solidification. For other cases the $\alpha$ parameter allow for taking into account solute diffusion which proceeds in solid phase at a finite rate, thus providing one with the so-called intermediate solidification models.

\section{Results of numerical simulation of the non-equilibrium solidification}

Computer simulations taking advantage of the calculation procedures of the ThermoCalc program and using the implemented Wołczyński solution are illustrated by the example of $\mathrm{Cr}-30 \% \mathrm{Ni}$ alloy. The model alloy was selected because due to wide range of solidification in region far from the eutectic reaction (Fig. 3). Moreover, it exhibits a single phase structure under the equilibrium conditions and is characterised by high solubility of nickel in the $\alpha$ solid solution. The mentioned features allow to illustrate clearly the influence of the degree of solute segregation on the solidification of the alloy.

Calculations were performed in the $P O L Y \_3$ module taking advantage of the SSOL database. This is a thermodynamic database built for the most popular metal alloys. The POLY_3 module is the main module of the ThermoCalc program, intended for calculation of phase equilibria by use of numerical methods. The module includes the iteration procedure named STEP, which in the NORMAL mode allows to follow the equilibrium solidification of the formerly selected binary or multicomponent alloy. The second available mode $(E V A L)$ serves for simulation of solidification proceeding in accordance with Scheil model. The STEP procedure combined with the EVAL mode was used to develop authors' own computer script intended for simulation of solidification with diffusion proceeding within the solid phase. The algorithm demands for the initial data such as: process parameters $(T, p)$, the initial chemical composition of the alloy, and the list of all phases which can occur in the solution. Then the liquidus temperature is determined for the alloy and the basic variable for the iteration procedure is defined. The core of the script is the calculation procedure (STEP) with implemented the transformed form of the model equation (1). Numerical solution proceeds in the following way: for every iteration step $\Delta T=1^{\circ} \mathrm{C}$, an increment in the solid phase $\Delta f_{S}$ and the solute concentration in the liquid phase are calculated on the basis of calculation of the momentary local equilibrium state. The obtained results of numerical simulation are presented in tabular form and/or in graphic form in the Post module.

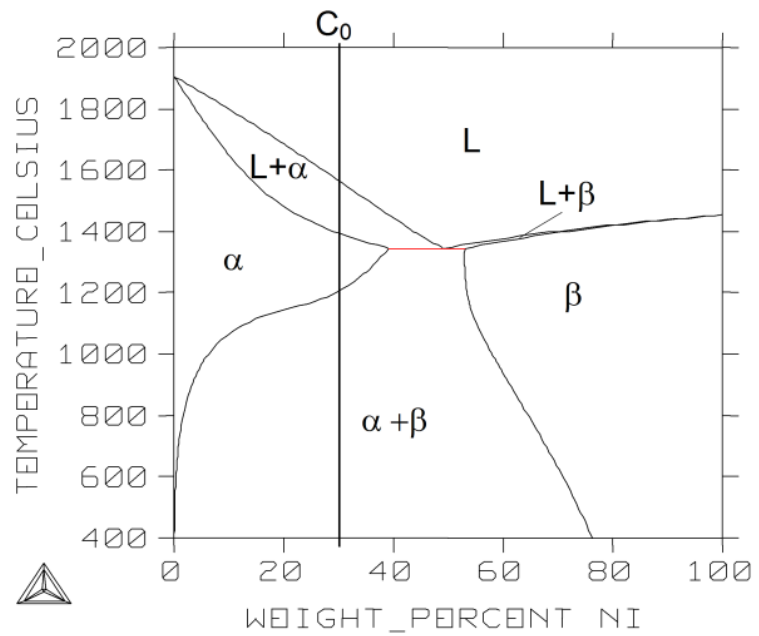

Fig. 3. Phase equilibrium diagram for $\mathrm{Cr}-\mathrm{Ni}$ system

Figures 4-7 show solidification curves, Ni-concentration profiles in the liquid phase during the solid phase growth, the change in the solidus temperature and the average solute concentration in the solid phase at the eutectic temperature for various values of back-diffusion parameter. The next figure (Fig. 8) depicts the change in phase composition of the alloy and the relationship between the quantity of the binary eutectics and the $\alpha$ parameter. The developed computational procedure takes also into account the change in the value of the equilibrium solute 
partition ratio as a function of temperature. The functional dependence $k_{0}(T)$ is presented in Fig. 9.

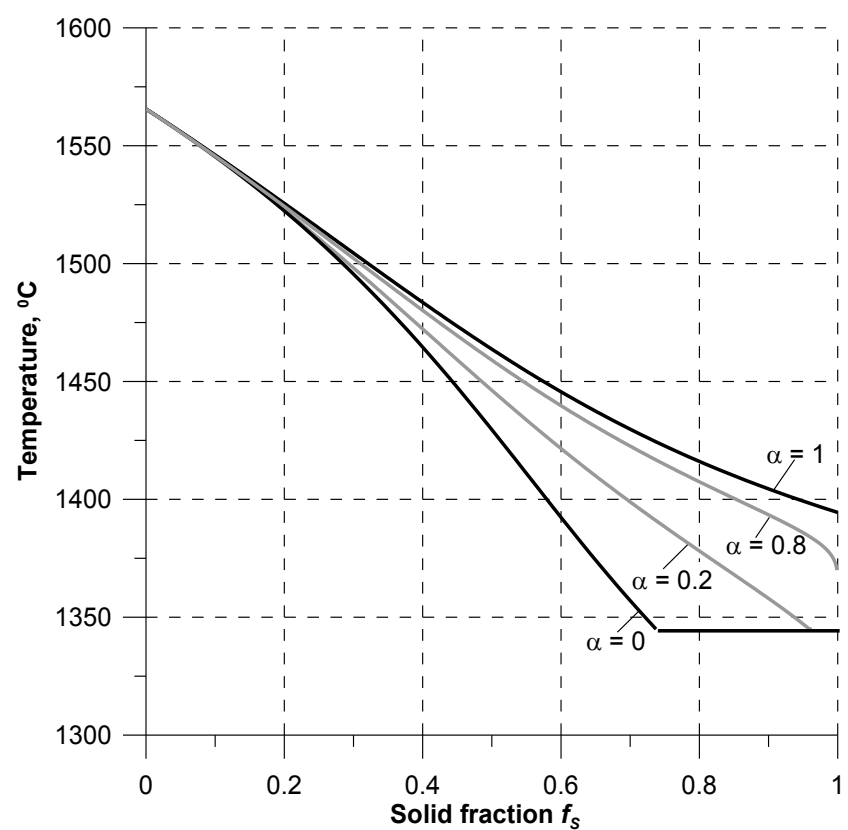

Fig. 4. The courses of solidification of $\mathrm{Cr}-30 \% \mathrm{Ni}$ alloy for various values of the back-diffusion parameter

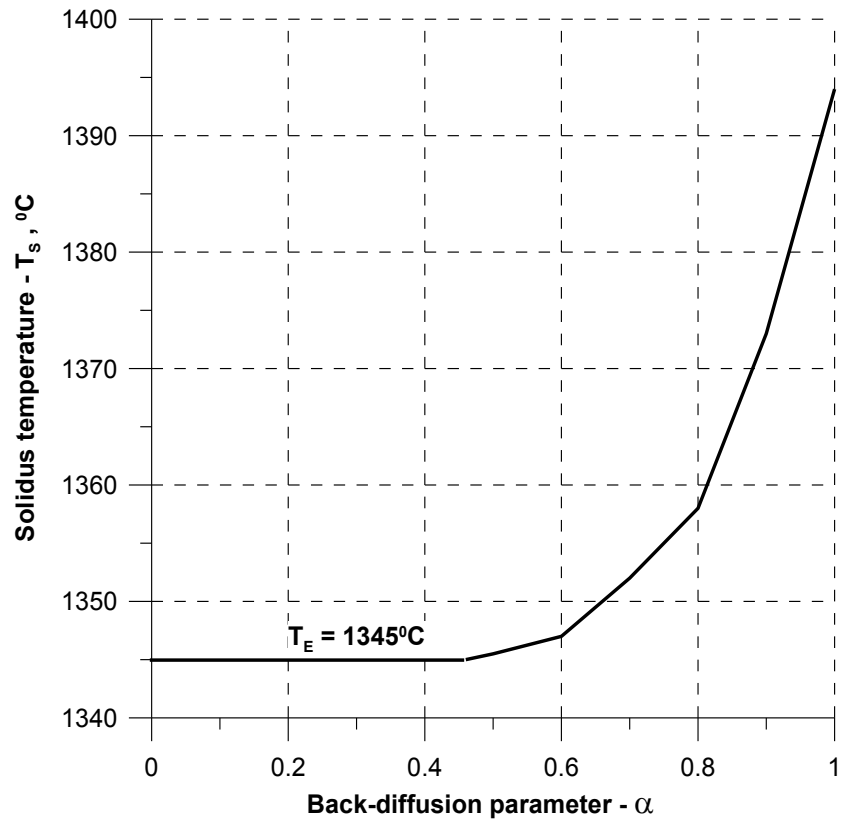

Fig. 5. The solidus temperature versus the value of back-diffusion parameter

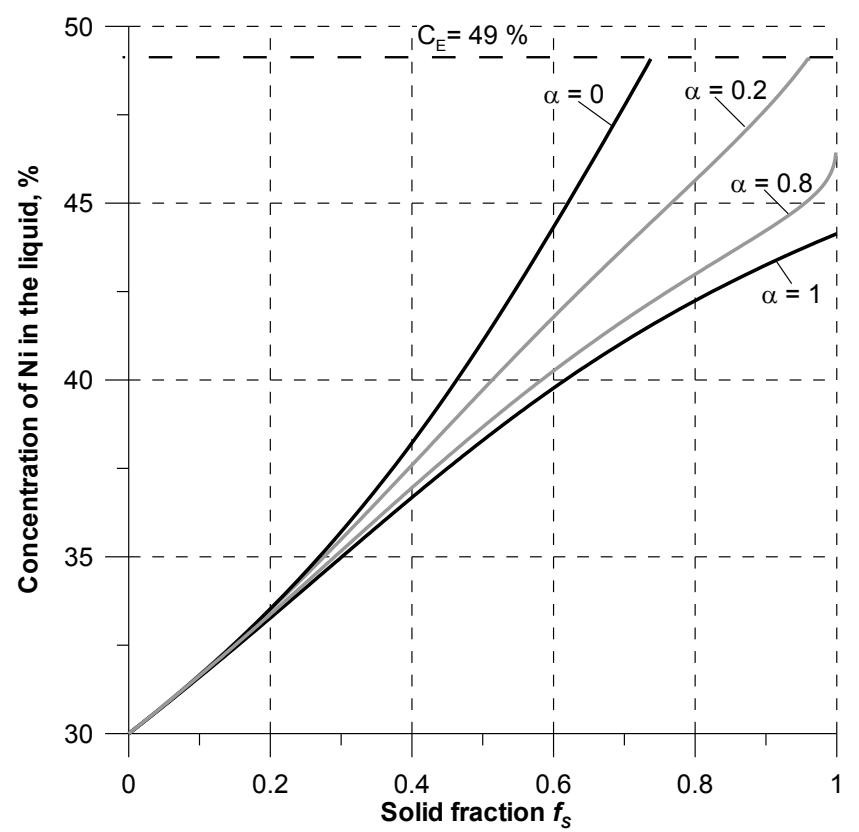

Fig. 6. Changes in nickel concentration in the liquid phase during solidification of $\mathrm{Cr}-30 \% \mathrm{Ni}$ alloy for various values of back-diffusion parameter



Fig. 7. The average Ni concentration in solid phase at the eutectic temperature for various values of back-diffusion parameter 


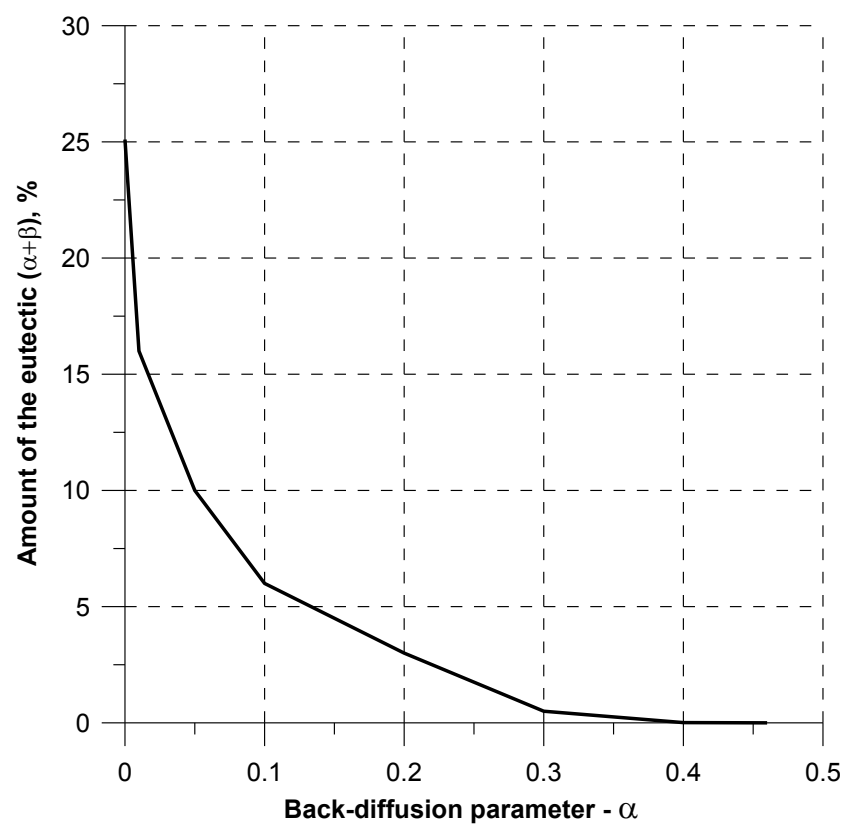

Fig. 8. Amount of the binary eutectics in $\mathrm{Cr}-30 \% \mathrm{Ni}$ alloy versus the value of back-diffusion parameter

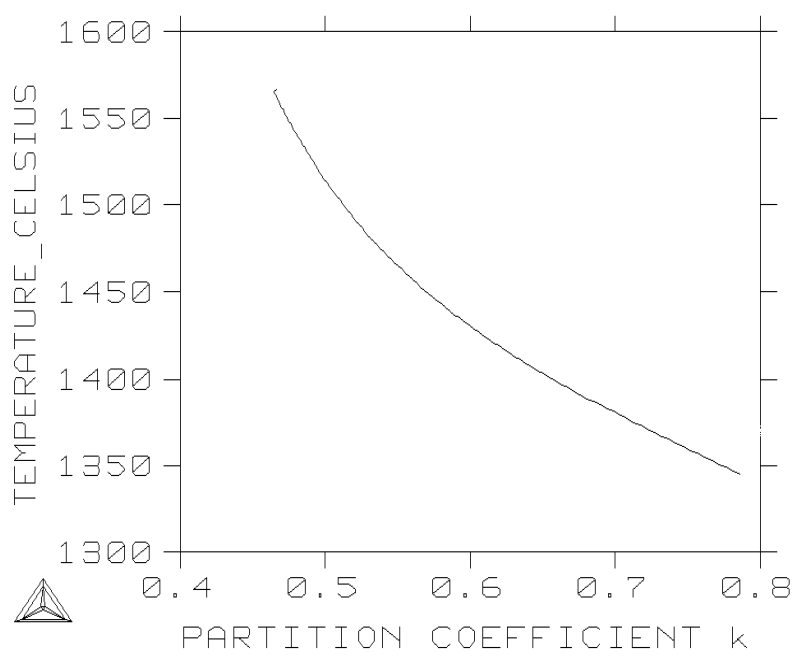

Fig. 9. Temperature versus the solute $(\mathrm{Ni})$ partition coefficient

Analysis of the calculation results indicate that the $\mathrm{Cr}-30 \% \mathrm{Ni}$ alloy under the equilibrium condition solidifies as a single phase material within the temperature range $1566^{\circ} \mathrm{C}-1394^{\circ} \mathrm{C}$. The phase composition of the alloy and the dependence between the solid phase fraction and temperature changes due to segregation of the alloying element, and the influence of these changes on the course of solidification depends on the value of the back-diffusion parameter (Fig. 4). The characteristic shown in Figure 5 indicates that as the $\alpha$ parameter decreases from 1 to 0.46 , the segregation of nickel results only in the change of solidus temperature, dropping gradually down towards the value of $T_{\mathrm{E}}=1345^{\circ} \mathrm{C}$. For the values of $\alpha$ less than 0.46 , the concentration of nickel in the liquid phase reaches $49 \%$ (Fig. 6) and the examined alloy, nominally the single phase one, solidifies with the eutectic transformation. The amount of eutectics rises with the decrease in the value of the $\alpha$ parameter (Fig. 8) and reaches 25\% under the solidification conditions corresponding to the diffusionless segregation $(\alpha=0)$. The average nickel concentration in the primary phase at the eutectic temperature falls between the values resulting from the equilibrium model and the Scheil model (Fig. 7).

The examined $\mathrm{Cr}-30 \% \mathrm{Ni}$ alloy is characterized by a strong change in the value of the partition coefficient as a function of temperature. The $k_{0}$ coefficient at the temperature of eutectic transformation is almost twice as large as at the temperature of the beginning of solidification. Many other models introduce linearization of the solidus and the liquidus curves while modelling the solidification process in order to assume the constant value of the $k_{0}$ coefficient. This simplification, however, results in large inaccuracy of the computational results in the case of many alloys solidifying in wide temperature ranges. The presented numerical procedure avoids such inaccuracies by calculation of the partition coefficient at every iteration step. The $k_{0}(T)$ dependence is illustrated in Figure 9.

\section{Final conclusions}

1. The developed computer executable script with implemented Wołczyński model allows to assess the course of the non-equilibrium solidification of binary alloys in the ThermoCalc program. The basic advantage of the applied numerical solution is that it can use large thermodynamic databases of the program and the iteration procedure available in the POLY 3 module, which is applied to determine the equilibrium state in binary or multicomponent systems.

2. Besides the possibilities of application of the ThermoCalc program in foundry technology described in the introduction, the application of the developed script allows additionally to find changes in the course of the alloy solidification depending on the degree of the alloying element segregation, which is expressed by the $\alpha$ parameter.

3. The numerical simulations make possible to trace the influence of the back-diffusion parameter on the profiles of the solidification curves, the solidus temperature, phase composition of the alloy and fractions of the individual phases, the solute concentration profile in liquid during the solidification, the average solute concentration in the solid phase at the eutectic temperature, and many other quantities which are usually calculated in the ThermoCalc program.

\section{References}

[1] Hillert, M. (1980). Empirical Methods of Predicting and Representing Thermodynamic Properties of Ternary Solution Phases. Calphad. 4, 1-12.

[2] Andersson, J.O., Fernández-Guillermet, A., Hillert, M. Jansson, B. \& Sundman, B. (1986). A compound-energy 
model of ordering in a phase with sites of different coordination numbers. Acta Metallurgica. 34, 437-445.

[3] Jansson, B., Schalin, M. \& Sundman, B. (1993). Thermodynamic calculations made easy. Journal of Phase Equilibria. 14, 557-562.

[4] Ansara, I. (2000). Models for composition dependence. Calphad. 24, 19-40.

[5] Sundman, B. Jansson, B. \& Andersson, J-O. (1985). The Thermo-Calc databank system. Calphad. 9, 153.

[6] Sundman, B. (1990). Review of alloys modelling. Anales de Fisic. Serie B. 86, 69-82.

[7] Hillert, M. (1998). Phase Equilibria, Phase Diagrams and Phase Transformations - Their Thermodynamic Basis. Cambridge University Press.

[8] Andersson, J.O., Helander, T., Hdghmd, L., Shi, P. \& Sundman, B. (2002). THERMO-CALC and DICTRA, Computational Tools For Materials Science. Calphad. 26, 273-312.

[9] Hillert, M., Höglund, L. \& Schalin, M. (2000). Computer Simulation of Cooling Curves for Solidification. Materials Transactions. 41, 1098-1103.

[10] Ohsasa, K. (2001). Numerical simulation of solidification for aluminum-base multicomponent alloy. Journal of Phase Equilibria. 22, 498-503.

[11] Hillert, M. \& Sundman, B. (1990). Scheil reaction scheme by computer. Calphad. 14, 111-114.

[12] Zyska, A., Konopka, Z. \& Łągiewka, M. (2006). Thermodynamic analysis of cast alloys by means of the ThermoCalc program. Archives of Foundry. 6, 531-536.
[13] Wróbel, M. \& Burbelko, A. (2014). CALPHAD method a modern technique of obtaining thermodynamical data. Archives of Foundry Engineering. 14, 79-84. (in Polish).

[14] Borgenstam, A., Höglund, L., Ågren, \& J. Engström, A. (2000). DICTRA, a tool for simulation of diffusional transformations in alloys. Journal of Phase Equilibria. 21, 269-280.

[15] Kobayashi, S. (1988). Solute Redistribution during Solidification with Diffusion in Solid Phase. A theoretical analysis. Journal of Crystal Growth. 88, 87-96.

[16] Nastac, L. \& Stefanescu, D. (1993). An analytical model for solute redistribution during solidification of planar, columnar, or equiaxed morphology. Metallurgical Transactions A. 24A, 2107-2118.

[17] Brody, H.B. \& Flemings, M.C. (1966). Solute redistribution in dendritic solidification. Transactions of the Metallurgical Society of AIME. 236, 615- 624.

[18] Wołczyński, W. (2000). Back-diffusion phenomenon during the crystal growth by the Bridgman method. Modeling of Transport Phenomena in Crystal Growth. Boston: WIT Press. 19-59.

[19] Kloch, J., Billia, B., Okane, T., Umeda, T. \& Wołczyński, W. (2000). Experimental Verification of the Solute Redistribution in Cellular/Dendritic Solidification of the Al3.5Li and Fe-4.34Ni Alloys. Materials Science Forum. 329330, 31-36.

[20] Fraś, E. (2003). Crystallization of metals. Warszawa: WNT. (in Polish). 